Supporting Information

\title{
Bioorthogonal metabolic labeling utilizing protein biosynthesis for dynamic visualization of non-enveloped enterovirus 71 infection
}

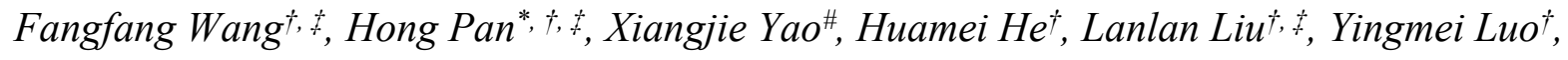

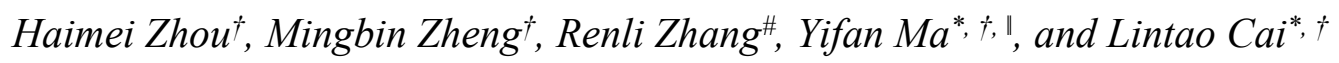

† Guangdong Key Laboratory of Nanomedicine, CAS-HK Joint Lab of Biomaterials, CAS Key Lab for Health Informatics, Shenzhen Engineering Laboratory of Nanomedicine and Nanoformulations, Shenzhen Institutes of Advanced Technology, Chinese Academy of Sciences, Shenzhen 518055, P. R. China.

‡ University of Chinese Academy of Sciences, Beijing, 100049, P.R. China.

" HRYZ Biotech Co., Shenzhen, 518057, P. R. China.

\# Shenzhen Centre for Disease Control and Prevention, Shenzhen 518100, P. R. China.

${ }^{*}$ Corresponding author:

*Email: 1t.cai@siat.ac.cn

*Email:yf.ma@siat.ac.cn

*Email:hong.pan@siat.ac.cn 
(A)

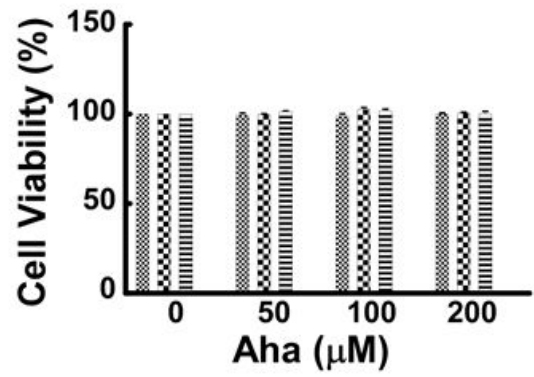

(C)

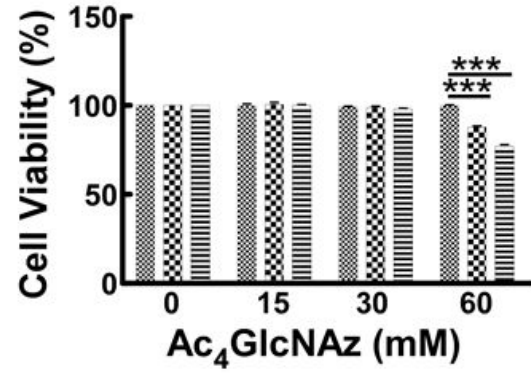

(B)
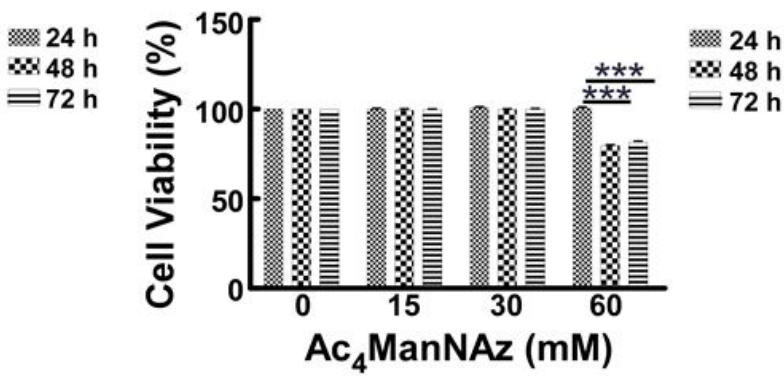

(D)
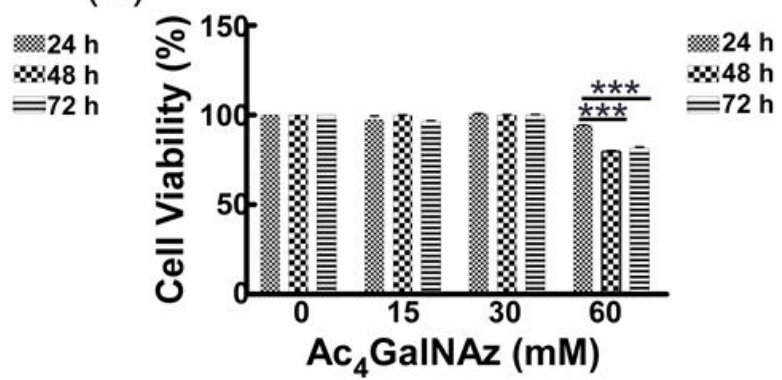

Figure S1. CCK8 assay analysis of the viability of RD cells treated with azido-amino acid or azido-sugars. (A-D) RD cells were incubated with $A$ ha, $A_{4}{ }_{4} M a n N A z, A c_{4} G l c N A z$, and $\mathrm{Ac}_{4} \mathrm{GalNAz}$ at indicated concentrations for $24 \mathrm{~h}, 48 \mathrm{~h}$, and $72 \mathrm{~h}$, respectively. The viability of RD cells was detected by Cell Counting Kit- 8 . Histograms display averages \pm SEM; $n=5$. There is no statistically significant difference in each group except the groups marked asterisks. **: $p<0.01 ; * * *: p<0.001$. 

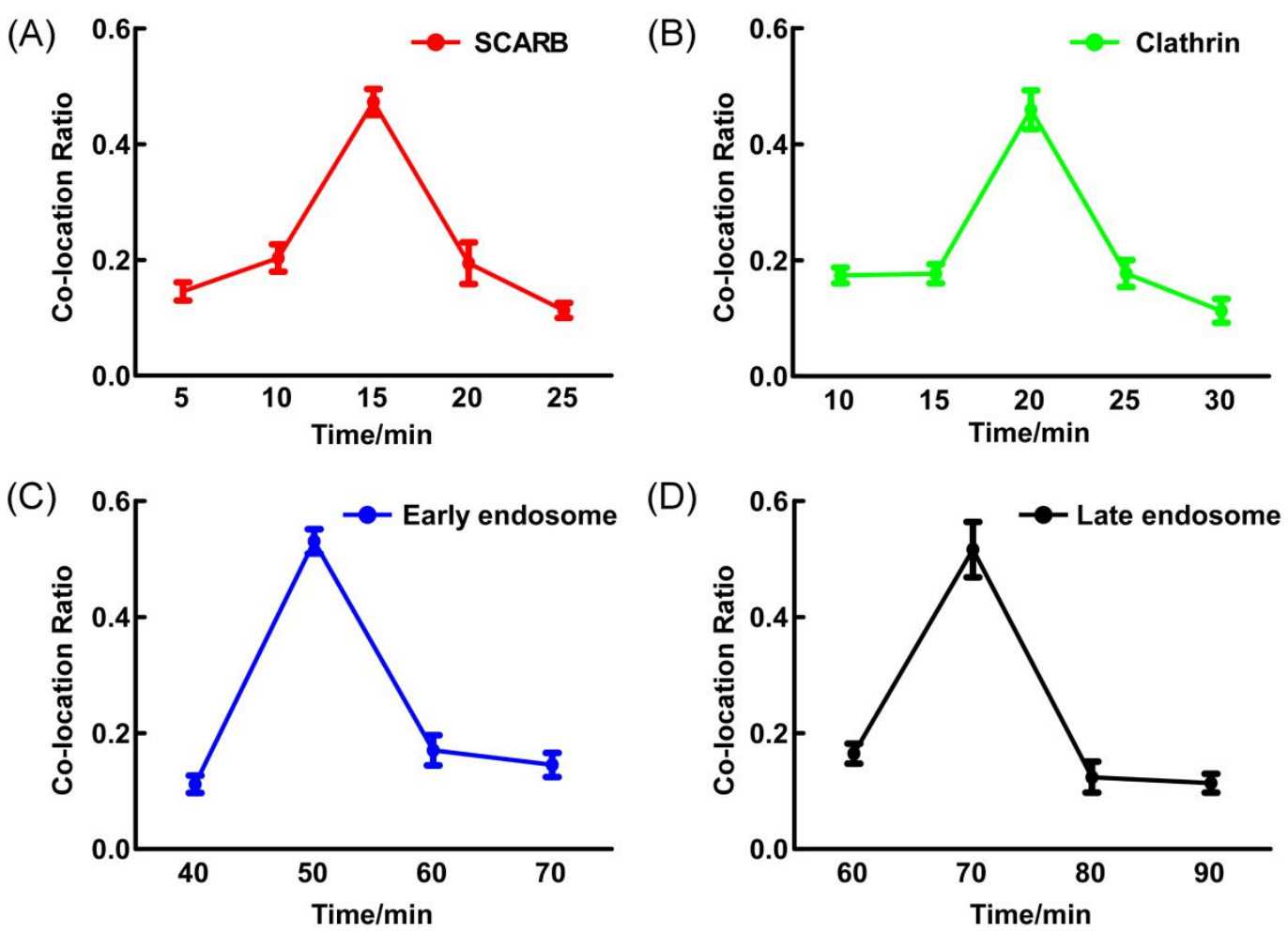

Figure S2. The mathematical statistics of time points of $\mathrm{N}_{3}$-EV71 particles in cellular organelles. The fluorescencent co-location ratio of EV71 was recorded and analyzed at each time point in different organelles, including (A) scavenger receptors, (B) clathrin, (C-D) early and late endosome.Number of colocalized cells in each group were counted over nine fields of view. Co-location ratio $=$ the numebr of colocalized cells / total number of cells in one field of view. 

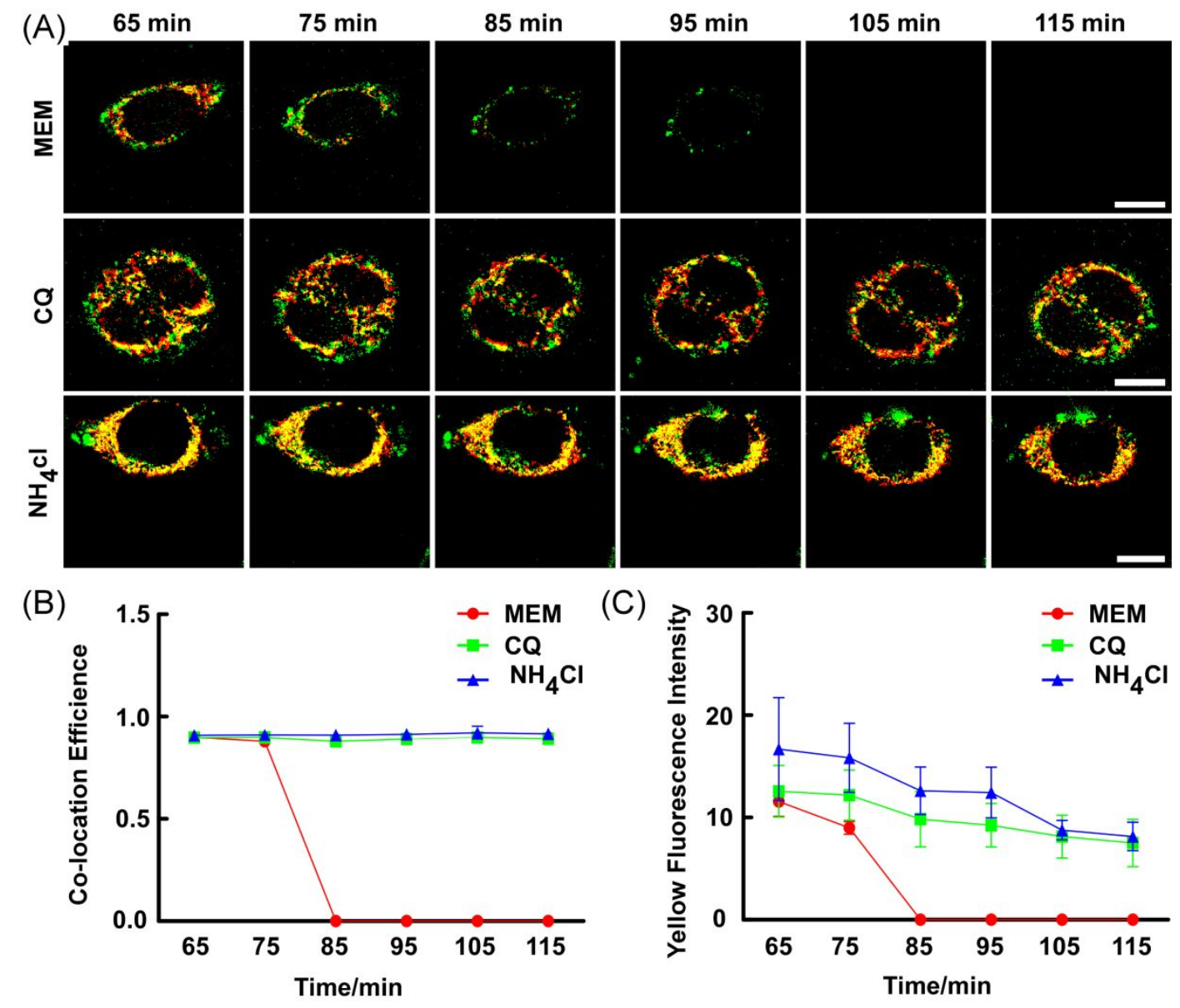

$115 \mathrm{~min}$

Figure S3. The effect of low-pH on EV71 uncoating in endosomes. (A) RD cells pretreated with $10 \mu \mathrm{M}$ chloroquine (CQ) or $100 \mu \mathrm{M} \mathrm{NH}_{4} \mathrm{Cl}$ were infected with dual-labeled EV71. (B-C) Co-location efficiency and intensity of yellow fluorescence. Scale bar $=5 \mu \mathrm{m}$. 
Movie S1. Live imaging of the result of DBCO-probes labeling on EV71. SYTO-82 (red) staining the viral RNA was shown as red and DBCO-Fluor488 was shown as green.

Movie S2. Live imaging of the process of in situ bioorthogonal labeling on $\mathrm{N}_{3}$-EV71 in host cells. The viral RNA was stained with SYTO-82 (red) and the azido motifs on viral capsids were probed by DBCO-Fluor488 (green).

Movie S3. Live imaging of the EV71 uncoating process. The viral RNA of $\mathrm{N}_{3}$-EV71 were stained with SYTO-82 (red), and the viral capsid was labeled with DBCO-Fluor488 (green). 